\title{
Validation of new superheavy elements and IUPAC-IUPAP joint working group
}

\author{
Cecilia Jarlskog a
}

Div. Math. Phys., Physics Department, LTH, Lund University, Sweden

\begin{abstract}
The great chemist Glenn Seaborg has written a delightful little book "Man-made Transuranium Elements", published in 1963, in which he points out that: "The former basic criterion for the discovery of a new element - namely, chemical identification and separation from all previously-known elements - had to be changed in the case of lawrencium (element 103). This also may be true for elements beyond lawrencium."

Indeed this is what has happened. The elements with $Z \geq 103$ are produced in nuclear reactions and are detected by counters. The detectors have undergone substantial refinement. For example one uses multiwire proportional chambers [for which George Charpak received the 1992 Nobel Prize in Physics] as well as solid state micro-strip detectors. In spite of this remarkable shift from chemistry to physics, the managerial staff of the International Union of Pure and Applied Chemistry (IUPAC) does not seem to be aware of what has been going on. The validation of superheavy elements should be done by physicists as the chemists lack the relevant competence as I will discuss here below.

This article is about a collaboration between International Union of Pure and Applied Chemistry (IUPAC) and its sister organization International Union of Pure and Applied Physics (IUPAP), to deal with discovery of superheavy elements beyond $Z=112$. I spent a great deal of time on this issue. In my opinion, the collaboration turned out to be a failure. For the sake of science, which should be our most important concern (and not politics), the rules for the future collaborations, if any, should be accurately defined and respected. The validation of new elements should be done by people who have the relevant competence - the physicists.
\end{abstract}

\section{Introduction in a nutshell: Physicists discover - IUPAC gets the credit}

The organizers of this Nobel Symposium have asked us, the speakers, to be

\section{"brave, simple \& bold"}

and that is what I intend to be, hoping for a better future.

My talk concerns the latest IUPAC-IUPAP Joint Working Group (JWG) which was created in 2011 to deal with issues concerning discoveries of superheavy elements beyond element 112. As the president-designate of IUPAP, and soon-to-become its president, I was asked to negotiate with IUPAC to establish the Group. Being naive and believing that scientists are impartial and logical I thought the task was going to be easy. I could not imagine that there would be "political" aspects in it. It turned out that the managerial staff of IUPAC

a e-mail: cecilia.jarlskog@matfys.lth.se

(C) The Authors, published by EDP Sciences. This is an Open Access article distributed under the terms of the Creative Commons Attribution License 4.0 (http://creativecommons.org/licenses/by/4.0/). 
cared primarily about getting credit rather than being fair and giving credit to those who deserved it. This behaviour is, in my opinion,

\section{old-fashioned, wrong, bad, and immoral.}

I wish to emphasize that I am not talking about the scientists, the chemists in IUPAC, but about their managerial staff.

After starting my work I realized that the responsibility put on my shoulders was heavier than I could have imagined. While discussing this matter in IUPAP, Alan Astbury (who served IUPAP for nine years, 2002-2011, as president-designate, president, and former president), in response to an email by me, sent the following lines in a message addressed to several of us (in September 2011, i.e., about two months before his appointment in IUPAP ended):

“... For historical reasons the Chemists cling to this process dearly. Judy and I spent hours on this, and look what we achieved!!"

Here Judy refers to Judy Franz who was the Executive Officer of the American Physical Society (APS). She was also the Secretary-General of IUPAP. Alan had a very high opinion of Judy and their collaboration was very successful. When I was asked to take charge of negotiations with IUPAC the office had already moved from APS to Institute of Physics (IOP) in London. People I consulted would tell me that chemists are "impossible to deal with" (especially the managerial staff of IUPAC, who were in addition arrogant and didn't answer emails). I simply could not believe this as I had met many chemists, for example at the Royal Swedish Academy of Sciences where I had been a member since 1984, i.e., by then for more than 27 years, and had found chemists to be just fine. So what was going on? It was clear that the roots of the problem were deep.

\section{Discoveries of superheavy elements fall into domain of nuclear physics}

Research papers on discovery of superheavy elements are almost all published in physics journals such as Physical Review Letters, Physical Review, Europhysics Journal, and so on. One may sometimes find a few articles in the Journal of Pure and Applied Chemistry, but these are in general not written by discoverers but by managerial staff of IUPAC or a working group reporting on what others have done.

The leading role of physicists in the discovery of the superheavy elements is not a new phenomenon. In 1951 the Nobel Prize in Chemistry was awarded jointly to Edwin Mattison McMillan and Glenn Theodore Seaborg. McMillan, who in 1940 had discovered the first transuranium element, declared in his Nobel Lecture:

"in spite of what the Nobel Committee may think, I am not a chemist."

He had used the cyclotron built by the 1939 Nobel Laureate in Physics, Ernest Orlando Lawrence, who was awarded the Prize

"for the invention and development of the cyclotron and for results obtained with it, especially with regard to artificial radioactive elements".

The Prize was also justified by the fact that

“... only with the cyclotron was it possible to produce active isotopes in large quantities. This was, among other things, an essential condition for the use of active elements for biological and medical purposes." 
Glenn Seaborg who succeeded McMillan, and was involved in discovery of about ten transuranium elements, described in his Nobel lecture the research environment at Berkeley as follows:

"The work on the transuranium elements which I am about to describe was made possible by this circumstance and because of the unusual and excellent spirit of cooperation which exists at the Radiation Laboratory, under the direction of Professor E.O. Lawrence, where the cyclotron bombardments so crucial to the final results were made."

In an interview Seaborg has said that he loved physics but became a chemist because there were more jobs for chemists than for physicists. The important point is that there was an admirable spirit of collaboration among these great scientists, irrespectively of which department they belonged to.

As the years passed, elements up to and including $Z=101$ were produced using neutron, deuteron, or He ions from the cyclotron, with the exception of $Z=99$ and $Z=100$, which were discovered in thermonuclear (bomb) explosions. Elements beyond $Z=101$ have been produced via collision of heavier projectiles such as carbon and calcium or zinc ions with transuranium elements or lead or bismuth, respectively. Seaborg expected transuranium elements to play an important role in energy production. However, this has not happened yet. Elements beyond $Z=100$ are produced in very small amounts in particle accelerators and decay rapidly. However, getting to know their properties is important for basic science.

\section{Why does it matter that the physicists get the credit they deserve?}

The area of superheavies is without any doubt very interesting. Physicists, whenever possible, try to study physics under extreme conditions hoping to learn something new and interesting about nature. That is why some profitless-looking enterprises are extremely interesting, such as studying black holes, even though we "pray" and hope that we will never meet any of them. Black holes constitute a laboratory for testing some fundamental ideas, such as the information paradox.

Superheavy elements fall into a more tangible extreme domain of physics. An obvious question is whether we will reach the so called, up to now hypothetical, island of stability, inhabited by long-lived superheavies, and if so what will we learn? I remember that many years ago there was a proposal called Lee-Wick vacuum, named after two outstanding theorists, T.D. Lee (Nobel Laureate in Physics, 1957) and his colleague Gian Carlo Wick. These authors were concerned with the structure of the "vacuum", the "nothing" which all along has enjoyed enormous popularity among theorists as this "nothing" may actually be something subtle. The familiar hypotheses of ether and of the Dirac sea, are two early versions of non-trivial vacua. In the Lee-Wick model, the "hyper-superheavies" were predicted to form not just an island but a continent. The nuclear matter was thus predicted to be highly "abnormal".

The superheavies, beyond element $Z=100$, that have been discovered up to now, are short-lived. However, they all have isotopes, many of them have been discovered but many more have not been accessible. Generally, one expects some neutron-rich isotopes to have much longer lifetimes and perhaps take us to the so called "island of stability". This makes the physics of the superheavies particularly interesting. Moreover, the decays of these objects involve a host of nuclear phenomena $(\alpha, \beta, \gamma$ decays as well as electron capture and spontaneous fission) that provide information about nuclei. The study of electronic structure 
of the superheavies is of interest to atomic physicists. However, these facts are not generally appreciated.

Nowadays, in several countries, the inhabitants are afraid of the word "nuclear". They do not realize that almost $100 \%$ of their own mass comes from the nuclei of hydrogen and heavier elements in their bodies. This fear has sometimes had ridiculous consequences. For example, in Sweden at hospitals the letter N in NMR (nuclear magnetic resonance) has been removed. One uses instead the acronym MR which people erroneously call "magnetic Röntgen", which means "magnetic X-ray". Another acronym used is MI (magnetic imaging). In fact research in nuclear physics is not rated highly. When the researchers apply for funding to produce and study superheavy elements, there is an eminent danger that the evaluators at funding agencies do not understand or appreciate their work and therefore discriminate against them. After all this field is rather "narrow" and cannot count on having supporters on the relevant panels. Superheavy hunters tend not to get the beam time that they deserve. In some cases, their project is canceled due to "funding priorities". Being bold I would add:

\section{It certainly does not help that in addition IUPAC steals credit from them!}

\section{IUPAC-IUPAP joint working group and what went wrong}

Some facts about early history of the IUPAC-IUPAP collaboration on transuranium elements can be found in a report by Barber et al. [Prog. Part. Nucl Phys., Vol. 29 (1992) pp. 453]. According to this report, the first attempt to form such a collaboration was made in 1974. A committee of nine people was appointed but according to the report "This committee never completed its work, nor issued a report nor, indeed, met as a group, though there were some correspondence between members". The next round started in 1985. I will not go into what happened but have to say, with great regret, that some of the leading scientists in this committee have already passed away, among them its chair Denys Wilkinson. From the statement made by Alan Astbury, that I quoted earlier [“... For historical reasons the Chemists cling to this process dearly. Judy and I spent hours on this, and look what we achieved!!'] I conclude that there were problems with the collaboration as recently as in 2011. Sadly, also Alan has passed away. In IUPAP, we have been particularly unfortunate when it comes to our history. We have had no permanent office anywhere. I have contacted a few people but nobody seems to know what was going on, further back in time than 1970s.

In 1987 a new Joint Committee was created which had seven members nominated by IUPAP [D.H. Wilkinson (UK) Chairman, R.C. Barber (Canada), A. Hrynkiewicz (Poland), M. Lefort (France), M. Sakai (Japan), I. Ulehla (Czechoslovakia), A.H. Wapstra (Netherlands)] and two nominated by IUPAC [N.N. Greenwood (UK), Y.P. Jeannin (France)]. The Committee took its task seriously and produced the Barber et al. report quoted above. Note that at that time none of these members was from a claimant country. This was supposed to be a rule to be followed. Unfortunately, this rule was violated later on.

Taking a leap in time, the 2011 IUPAP General Assembly took place in London, 2-4 November. Two documents on IUPAC-IUPAP collaboration were presented to the delegates at this meeting. One was the request from the Inter-Union Group that the General Assembly approve the names and symbols for elements 110, 111 and 112. The second document was called Joint IUPAC/IUPAP Working Groups to Validate Claims for the Discovery of New Elements. It was information on Guidelines for the Appointment of Members as well as a list of names of the nominees from IUPAC and IUPAP. In retrospect, this document was already obsolete when it was presented. 
The IUPAP members of the latest Joint Working Group were suggested by the IUPAP Commission C12 - Nuclear Physics, at their annual meeting at MIT on 25 July 2011! IUPAP followed their recommendation as the Nuclear Physics Commission is the only one in IUPAP which has the relevant competence. It would have been "silly" if such a recommendation had come from the managerial staff! The guidelines included the statements that the members should not come from a claimant country and that each Union be represented by at least two members. And then just about everything went wrong.

Committing errors is said to be human, but repeating them is stupid. Instead one should learn from them, in order to avoid them. When charged with the above duty I took for granted that the two Unions will collaborate on all issues having to do with the discovery of superheavy nuclei, i.e., also on validations, and making announcements. Everyone agrees that the discovery of the new superheavy elements, a process which takes ages, lies in the "camp of physicists". Once discovered, the atomic physicists and chemists can deal with the identification of the electronic structure of these elements. As noted earlier, our physicists deserve and need to be publicly acknowledged for their achievements. However, it has turned out that the IUPAC managerial staff has gone behind our back and violated the "ethical rules", in spite of not having the competence to validate the discoveries.

In connection with the latest announcements by IUPAC, it is said that IUPAC collected referee reports but lacking the sufficient competence how can you choose appropriate referees and how can you evaluate their reports? We in IUPAP have been kept out of this process; we have not seen these reports. However, I know from private sources that they were not all positive. A major idea was to avoid having members from claimant countries (Germany, Japan, Russia, and USA). This was not followed. In fact IUPAC took the liberty of proposing a chemist from the USA as the chair! This worried me. I contacted Alan Astbury who wrote back to me "I presume he [meaning the Chair] feels that being God-like he can rise above all conflicts." This turned out to be very unfortunate. I should have protested. I was too naive to take for granted that the two sister Unions would operate together, in the spirit of heroes of the past such as Lawrence and Seaborg. The Chair was reluctant to communicate. As an example, at the end of his report to the 2014 IUPAP General Assembly in Singapore (which was supposed to be on behalf of the Joint Working Group) he had added:

"Disclosure: This informal progress statement has been prepared entirely by the Chair of the JWP and has not been reviewed by the membership."

I do not know what was going on. The great surprise was that IUPAC, unexpectedly and without consultation with us, released the results of "our" Joint Working Group on 30 December 2015 (note the date!). On that occasion one of the members of the Working Group sent me the following message:

“... I know you are now Past President of IUPAP, but I am writing because we corresponded about the IUPAP/IUPAC Joint Working Group in the past. As you know our reports on elements 113, 115, 117, and 118 have been accepted for publication in Pure and Applied Chemistry as is the case for past reports. Yesterday IUPAC released a press release, which I have attached. I am just letting you know in case there is anything IUPAP should do. My apologies in case I should have done anything sooner to let you know this was happening. I was not sure of the timing of the publication and thought it was going to be next year. I also had assumed the IUPAC and IUPAP were working jointly on this and there might be a joint announcement. ..." [my bold-facing]. 
This is not a good sign. It only shows that there has been something fundamentally wrong in this Inter-Union Collaboration. Not even (all) the members of the committee were informed about what was going on!

\section{Looking ahead}

Times have changed and the old rules and guidelines are no longer appropriate. As Seaborg predicted, more than 50 years ago, the former basic criterion for the discovery of superheavy elements moved a long time ago from chemistry to physics. Superheavies are created by physicists at accelerators and are identified by using physical measuring devices. Only physicists have the competence to check the validity of the results. It is natural that IUPAP should take the leading role in this enterprise.

Another point of concern is that the managerial staff of IUPAC as well as IUPAP keep on changing. Even if say the two presidents would happen to know each other well and trust each other their terms are limited to two years in IUPAC and three years in IUPAP. The superheavies take much longer time to discover. Moreover, the presidents, and other managerial staff, should humbly be aware of the fact that they do not have the competence to pass judgment on superheavies.

We all hope that new superheavy elements will be found. If so, IUPAC and IUPAP may decide to form a new Joint Working Group, even though the contribution of IUPAC cannot possibly be significant. IUPAP, through its Commissions C2 (Commission on Symbols, Units, Nomenclature, Atomic Masses and Fundamental Constants) and specially C12 (Nuclear Physics) possesses sufficient competence to deal with the most important aspects of these discoveries. Since these elements are expected to have very short lifetimes, the determination of their electronic structure could be a challenge.

In my opinion, if there is going to be a new Joint Working Group, it is essential that IUPAP should insist on a well-defined mandate for the group. IUPAP should insist on openness. The referee reports should be ordered by IUPAP and be available for scrutiny by eligible nuclear physicists.

It is irritating when IUPAC "proudly announces" the discovery of new elements. What have they done to be proud of? They should congratulate the discoverers. You should not steal someone's discovery and be proud of it. The articles in the non-scientific journals do not help either. They give the credit to IUPAC, by making statements such as "The International Union of Pure and Applied Chemistry, which verified their existence, announced the entry of the four elements ....'. Well, I am sorry to have to say that those who made the announcement did not have the competence to verify "their existence".

First of all, I wish to thank the organizers of this Nobel Symposium for giving me the opportunity to be "brave, simple \& bold". I wish to thank Prof. Dan-Olof Riska who was the Chair of the Nuclear Physics Commission of IUPAP, 2008-2011, and his successor Prof. Hideyuki Sakai. Dan-Olof, with whom I had an extensive email exchange, answered all my questions and ensured that his commission, C12, efficiently proposed members to be included in the Joint Working Group. Hide-san remained in constant contact with me. On this occasion, my thoughts go to the late Alan Astbury, a brave \& bold man who truly cared for IUPAP. I wished I could have expressed my deep sense of gratitude to him. Finally, and above all, I wish a successful future for the superheavy community. 